\title{
Die Umsetzung des Vereinbarten
}

Die Bedeutung und der Ablauf der Umsetzung des Vereinbarten hängen sehr stark von der Art des Vertrags ab. Gerade bei komplexen Großprojekten, wie Bauprojekten, Anlagenbauverträgen etc., stellt die Vertragsumsetzung eine große Herausforderung dar. In solchen Fällen ist die Parteibeziehung ( $\rightarrow$ Beziehungsebene) von besonderer Bedeutung, da es hier häufig auf die Kooperation der Parteien in der Umsetzungsphase ankommt. Die Verhandlungspartner bedürfen in solchen Fällen $\rightarrow$ Vertrauen ineinander. Das Risiko von Streitigkeiten ( $\rightarrow$ conflict aftermath) ist bei solchen großen Projekten sehr hoch, weshalb ex ante Regelungen für mögliche Eskalationen geschaffen werden sollten (z. B. $\rightarrow$ Eskalationsstufen). Die Vertragsdurchführung müssen die Verhandler bereits in allen vorgelagerten Verhandlungsphasen im Blick behalten. Insbesondere $\rightarrow$ realmaker fokussieren sich auf die reibungslose Vertragsdurchführung und versuchen diese, durch umfassende Regelungen im Vertrag ( $\rightarrow$ safe harbor principle) sicherzustellen. Es lassen sich jedoch ex ante nicht alle eventuell eintretenden zukünftigen Situationen regeln ( $\rightarrow$ unvollständige Verträge). Mit Hilfe des $\rightarrow$ legal risk management sollten rechtliche Risiken - auch solche, die im Rahmen der Vertragsdurchführung auftreten - analysiert werden.

Die Stichworte in diesem Werk beschäftigen sich nur am Rande mit der Umsetzungsphase. Zentraler Bestandteil der Umsetzungsphase ist das sog. $\rightarrow$ Claim-Management. Dabei geht es um das Erkennen von Abweichungen des Ist-Zustandes vom vertraglich vereinbarten Soll-Zustand und die Durchsetzung daraus entstehender Ansprüche sowie die Verhinderung der Entstehung oder Durchsetzung der Ansprüche des Vertragspartners.

$\rightarrow$ Nachverhandlungen sind ebenfalls ein typischer Bestandteil der Umsetzungsphase. Solche Nachverhandlungen können legitim sein, wenn sich Umstände geändert haben. Sie können sogar dazu genutzt werden, den Verhandlungskuchen ( $\rightarrow$ negotiation pie) zu vergrößern und somit ein besseres Ergebnis für beide Seiten zu erzielen. Problematisch sind dagegen opportunistische Nachverhandlungen ( $\rightarrow$ Nachverhandlungen). 